\title{
Cucurbitacins and its Anticancer property: A Review
}

\author{
Available online at www.hjhs.co.in \\ REVIEW ARTICLE \\ Ashmi samuel ${ }^{\text {a }}$ \\ ${ }^{a}$ Dr Joseph Mar Thoma Institute of Pharmaceutical Sciences and Research Dist, Pallickal, P.O, \\ Kayamkulam, Kerala, India 690503. \\ DOI 10.22270/hjhs.v4i4.46
}

\begin{abstract}
Cucurbitacins which are structurally unlike triterpenes found in the members of Cucurbitaceae and several other plant families possess immense pharmacological activity. This diverse group of compounds may substantiate to be important lead molecules for the treatment of cancer. Research focused on this unattended medicinal compound can prove the significance and explore the hidden potential to cure various diseases. These compounds show their proposed mode of action, probable molecular targets and have future aspects of their use as a medicinal agent. This review is aimed to provide the chemical nature and medicinal property of cucurbitacins.
\end{abstract}

Keywords: Cucurbitacin, STAT, Janus kinase (JAK), anti-tumor, anti-inflammation, apoptosis.

\section{Introduction}

Medicinal plants are the nature's gift to human being to cure disease and make disease free life. Herbal medicine is still the backbone of about $75-80 \%$ of the total population (Ali MS et al., 2018). Plant's secondary metabolites are important sources for scientific and clinical study as well as for new drug development. Many plant synthesized herbal medicines in different formulations used for treatment of various diseases from an ancient time. Traditional or indigenous drugs have special importance. Phytochemicals as secondary metabolites and they can be used in the pharmaceutical industries for producing a potent drug (Nag M et al., 2018). Terpenes are volatile organic compounds which are the major odoriferous constituents of essential oil and contain two or more 5-carbon isoprene units. Many terpenes contain additional functioning groups, such as carboxyl $(\mathrm{COOH})$, carbonyl (CO), alkoxy (OR) and hydroxyl $(\mathrm{OH})$. It is tested over a long time, and is relatively safe, easily available and affordable (Kupchan, Meshulam, \& Sneden, 1978). Many ethno-botanical survey reported that, the local population in different parts of the world including the USA, China, India, Mexico, Morocco, Saudi Arabia, Taiwan, and Trinidad and Tobago depend on medicinal plant and suggested that several medicinal plants have been used as dietary adjuncts for the cure of numerous chronic and severe disease $(\mathrm{H}$ Stuppner \& Moller, 1993). In India and China, the use of herbal medicines has been commonly used from a long time, as a less expensive way to treat various health problems. The herbal drugs are less toxic with limited side effects compared with synthetic drugs (DINAN et al., 1997). Due to such reasons, traditional and complementary medicines have seen an increase in their popularity for the treatment of different diseases. The WHO has also recommended the initiation of studies to identify and characterize new herbal preparations from traditionally known plants and the development of new effective therapeutic agents, especially in the areas where the lack of safe modern drugs to treat chronic diseases. Cancer is a chronic disease and serious health threats for about seven million people in a year and a big challenge medical science (Makar, Saha, \& Singh, 2018). In the ongoing search for more effective and safer drugs, attention is being paid to new and safe medicinal herbs or food components. Although phyto-therapy continues to be used in several countries as in the past, only a few plants have received scientific or medical scrutiny (Kaushik, Aeri, $\&$ Mir, 2015). Although most of the herbal plants are safer, still a number of medicinal plants possess some degree of toxicity; 
therefore it is very important to analyze the traditional therapeutic regimens scientifically and their dosing, toxicity and other health consequences, before proper use in human diseased conditions. Now a days the environment becomes highly polluted with variety of toxic chemical and waste biological active substances such as dyes, polymers, steroids, phytochromes, or the ubiquitous free radicals (Dinan, Harmatha, \& Lafont, 2001). Cucurbitacin are terpenoids category of diverse compounds found in the plants of family Cucurbitaceae. These compounds have medicinal and toxic properties. These compounds are also discovered in other plant families like Scrophulariaceae, Cruciferae, Datiscaceae, Primulaceae, Rubiaceae etc. The diversity of Cucurbitacin lies in variety of its side chain derivatives that contribute to their disparate pharmacological actions. The bitter taste of plant species like cucumber has been identified to the presence of Cucurbitacin. The first Cucurbitacin was isolated as a crystalline form substance in 1831 and was named $\alpha$ elaterin (Gamlath, Gunatilaka, Alvi, \& Balasubramaniam, 1988).

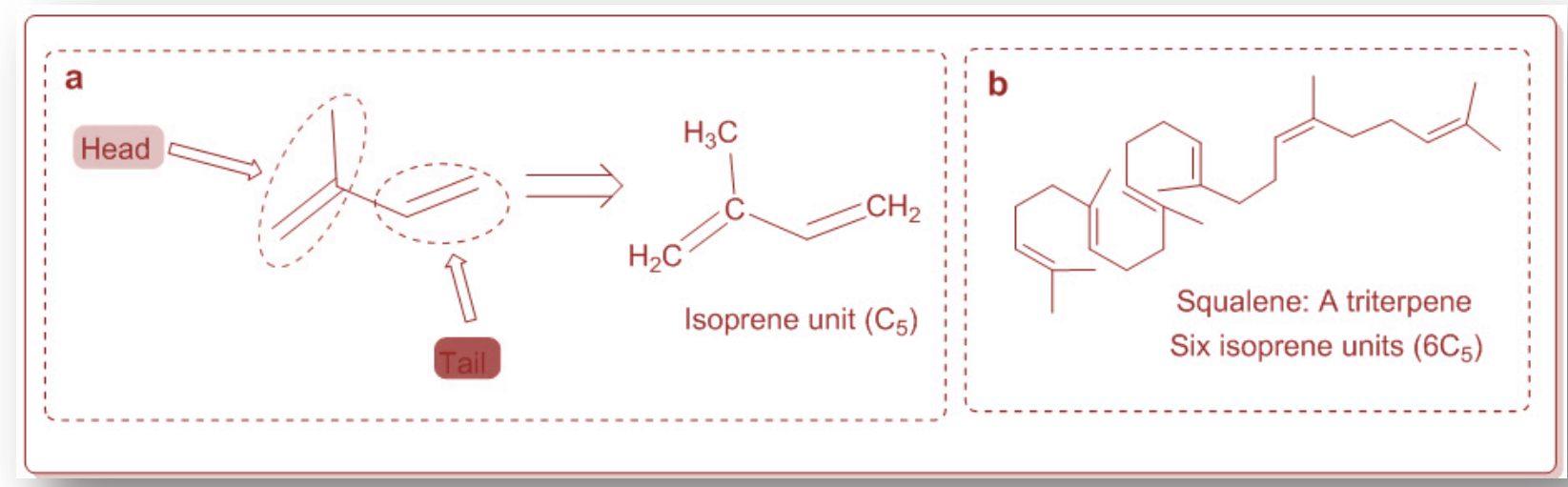

Figure. 1 (a) Isoprene unit and (b) triterpene (Squalene)

\section{Occurrence}

Cucurbitacins are found in many cucurbitaceous plants. They are most common in species of the Bryonia, Cucumis, Cucurbita, Luffa, Echinocystis, Lagenaria and Citrullus. The plants of genera Momordica contain a special group of Cucurbitacins called momordicosides. The level of Cucurbitacins varies between tissues. They may be concentrated in fruits and roots of mature plants. In fruits where Cucurbitacins are produced, their highest concentration is achieved on maturity. Seeds generally contain very low concentration of Cucurbitacins.

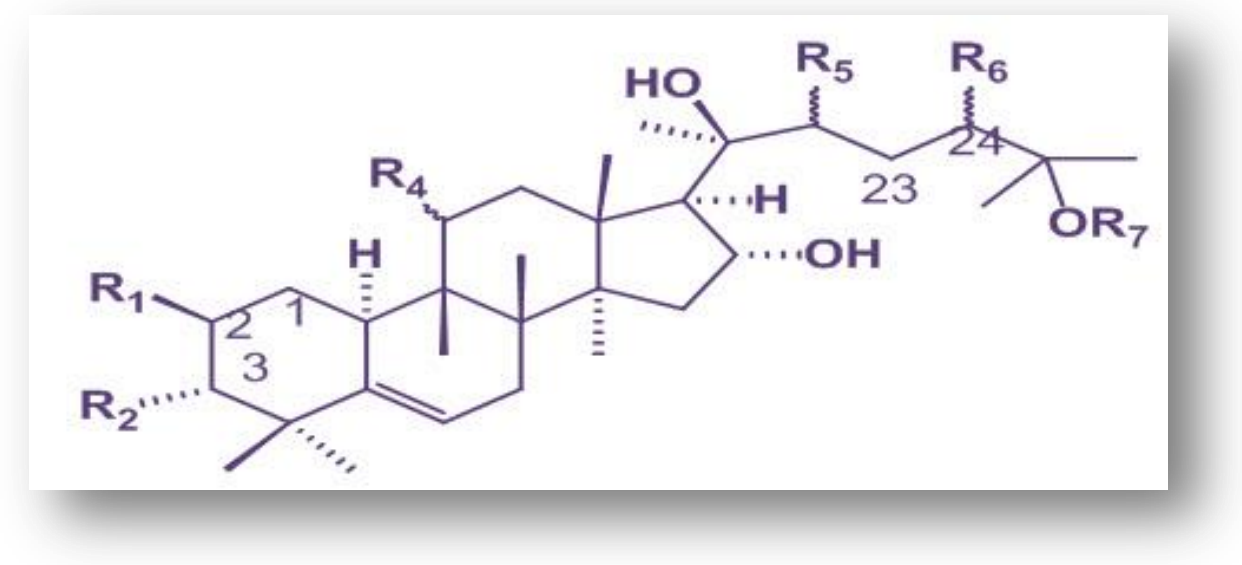

Figure.2 General structure of Cucurbitacin (Gry, 2006) 
Cucurbitacin producing plants have also been identified outside the cucurbitaceae in the members of Scrophulariaceae, Begoniaceae, Primulaceae, Liliaceae, Tropaeolaceae and Rosaceae. The seeds of certain cruciferous plants, like Iberis species and Lepidium sativum also contain cucurbitacins. It is reported that Cucurbitacins are formed in situ and are not transported to another parts of the plant. The distribution of Cucurbitacins among several families of plant kingdom (Hermann Stuppner, Müller, \& Wagner, 1991).

\section{Chemistry of Cucurbitacins}

Cucurbitacins are a group of highly oxygenated triterpenoid molecules found in diverse species of cucurbitaceae family (Zheng, Fu, \& Pei, 2007). They are derived from the cucurbitane scaffold. That are widely present intraditional Chinese medicines, possess strong anticancer activity, andare divided into 12 classes from $\mathrm{A}$ to $\mathrm{T}$ with over 200 derivatives. All cucurbitacins contain a basic $\quad 19-(10 \rightarrow 9 \beta)-$ abeo--10 $\alpha$-lanost-5--ene ring skeleton. A common feature among all compounds in the category of Cucurbitacins is the presence of 5, (6)-double bond. The difference of Cucurbitacins from steroidal moiety lies in the fact that in basic structure of Cucurbitacins a methyl group is located at C-9 rather than C-10. Most of the Cucurbitacins are tetracyclic, but some representatives have an additional ring due to formal cyclization between C-16 and C-24 as in cucurbitacins S and $\mathrm{T}$. The Cucurbitacins differ from most of the other tetracyclic triterpenes by being highly unsaturated and contains numerous keto, hydroxyl, and acetoxy groups. Certain Cucurbitacins have been discovered in the form of glycosides and some of them lack C11 carbonyl function. Chemically, Cucurbitacins are ranked according to presence of various functional groups on rings $\mathrm{A}$ and $\mathrm{C}$, diversity in side chain and stereo chemical considerations. The structural composition of following Cucurbitacins are known and have been designated by the letters: A, B, C, D, E, F, G, H, I, J, K, L, O, P, Q, R and S. The term "Cucurbitacin" refers to group of
Cucurbitacins along with their glyosidic forms mentioned above. Cucurbitacin $\mathrm{G}$ and $\mathrm{H}$ have same structures but differ from each other in the configuration of the hydroxyl group at position 24 which is not yet established. Cucurbitacin $\mathrm{R}$ was demonstrated to be 23, 24-dihydrocucurbitacin D (DHCD) hence, its description has been moved to the group of Cucurbitacin D. Similarly Cucurbitacin J and $\mathrm{K}$ differ from each other only in the configuration of hydroxyl group at position 24 which is yet to be determined. A special group of Cucurbitacins are called as momordicosides, named after their occurrence in Momordica charantia. Momordicosides have never been identified in any other plant species. The common feature of momordicosides is that C19 has been oxidized to an aldehyde group.

The saccharide linkage is generally present at C-2 (2-O- $\beta$-glycosides) in Cucurbitacin glycosides. Majority of Cucurbitacins are usually crystalline in nature or present in the form of needles at room temperature except Cucurbitacin $\mathrm{H}$ which is an amorphous solid. Most Cucurbitacins are soluble in petroleum ether, chloroform, benzene, ethyl acetate, methanol and ethanol, but are insoluble in ether. They are only slightly soluble in water. Cucurbitacins usually have absorption maxima for ultraviolet light wavelength $(\lambda)$ between 228-234 nm (J. C. Chen, Chiu, Nie, Cordell, \& Qiu, 2005). Nine cucumber genes are also responsible for biosynthesis of cucurbitacins and elucidated four catalytic steps.

The transcription factors Bl (Bitter leaf) and Bt (Bitter fruit) that control this pathway in leaves and fruits, respectively. The $\mathrm{Bi}$ gene confers bitterness to the entire plant and is genetically related with an operon-like gene cluster, similar to the gene cluster involved in thalianol biosynthesis in Arabidopsis. Fruit bitterness requires both $\mathrm{Bi}$ and the dominant $\mathrm{Bt}$ (Bitter fruit) gene. Non bitterness of cultivated cucumber fruit is conferred by bt, an allele selected during domestication. $\mathrm{Bi}$ is a member of the oxidosqualene cyclase (OSC) gene family. 
Table. 1 Structures of Cucurbitacins

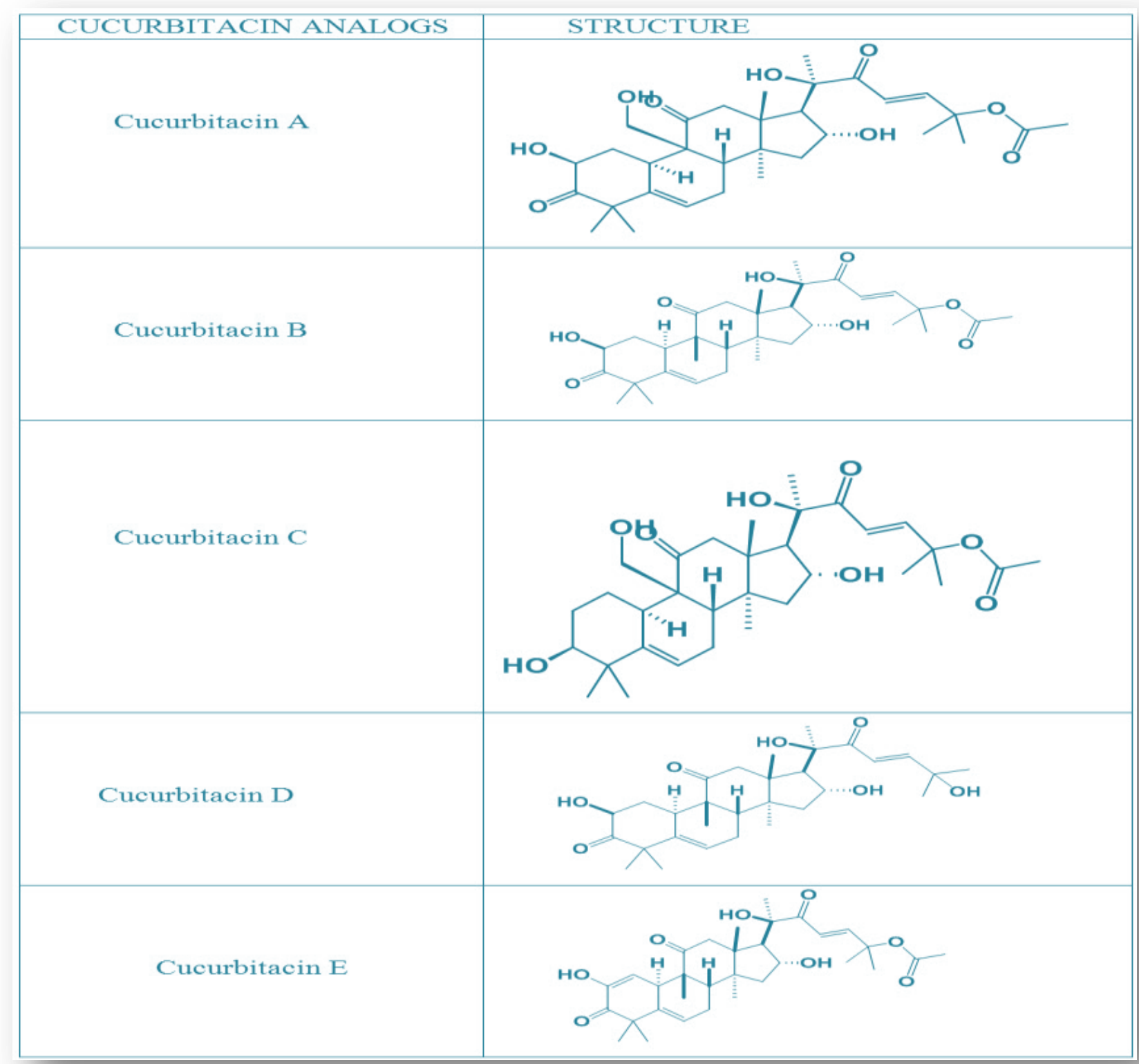

\section{Anticancer activity of Cucurbitacin and its derivatives}

Cancer is a rapidly growing health threat. Cancer is a group of diseases in which the abnormal growth of cells with the potential spread to other parts of the body. Not all tumors are cancerous in the starting phase and do not spread to other parts of the body. The sign and symptoms include a lump, abnormal bleeding, prolonged cough, unexplained weight loss etc. these symptoms may indicate cancer (Kaushik et al., 2015). The cancer is a serious diseases cause 8.8 million people worldwide died from cancer in 2015. That is nearly 1 in 6 of all global deaths. Only $5-10 \%$ of all cancer cases can be attributed to genetic defects, whereas the remaining 90-95\% has their roots in the environment and life style. The life style factors include cigarette smoking, diet, alcohol, sun exposure, environmental pollutants, infections, stress, obesity, and physical in activity (Teuscher, 1994). The evidence indicates that of all cancer related deaths, almost $25-30 \%$ are due to tobacco, as many as $30-35 \%$ are linked to diet, about $15-20 \%$ are due to infections, and the remaining percentage are due to other factors like radiation, stress, physical activity, 
environmental pollutants (Enslin, Joubert, \& Rehm, 1956). It presents the biggest challenge to researchers and medical professionals and has been selected for various prevention and therapeutic strategies. The dietary intake of many vegetables and fruits has been found to reduce the risk of occurrence of cancer. Cancer is the deregulation of apoptosis that programmed cell death. Several promising targets for intervention is identified by studying the molecular abnormalities that tumor genesis, such as the signal transduction pathways that regulate apoptosis. In this perspective, cucurbitacins and its derivatives have become a new focus for cancer treatment because of their strong capability to inhibit several cancer. The target area of cucurbitacins and its derivatives through JAK/STAT pathway, Wnt signaling and MAPK pathway (Njoroge \& Newton, 1994). Cucurbitacin and its derivatives are inhibited cancer growth via a wide range of mechanisms, including proapoptosis, induction of autophagy, cell cycle arrest, inhibition of cancer invasion, and migration. Cucurbitacins also modulate multiple intracellular signaling pathways.

It is interesting that each variant of cucurbitacins may trigger slightly different molecular signaling cascades in different cancer cell types to inhibit cancer growth and progress (Lavie, Willner, \& Merenlender, 1964). Signal transducers and activators of transcription 3 (STAT3) and Janus kinase (JAK) signaling pathways are the main mechanisms for cucurbitacins to induce apoptosis to exert their potent anticancer effect. In addition, the ability of cucurbitacins to induce cell cycle arrest in $\mathrm{G} 2 / \mathrm{M}$ phase mediated by various regulators is also an important way to fight against various cancers (Bartalis \& Halaweish, 2005).

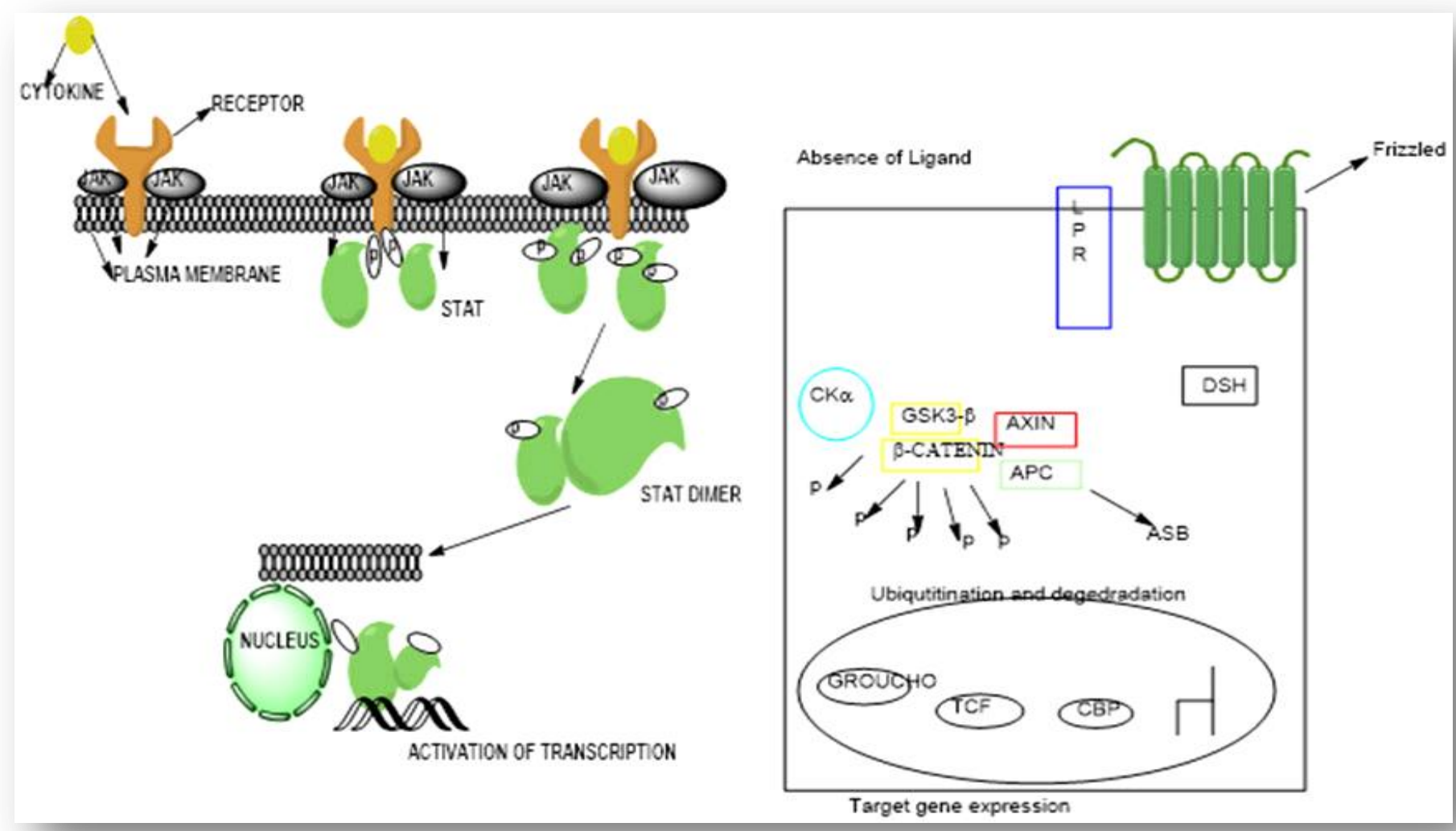

Figure. 3 (a) Mechanism of STAT signaling pathway leading to activation (Bowman, Yu, Sebti, Dalton, \& Jove, 1999) (b) Canonical and noncanonical Wnt signaling pathway

Cancer is responsible for $12 \%$ of the world's mortality. Treatments include surgery, and radio- and/or chemo-therapy. However, chemotherapy suffers limitations of side- effects, toxicity and drug resistance. Also, most established chemotherapy drugs are lacking specificity toward tumor cells.(1) Therefore, there has been a growing interest in 
the use of herbs as a promising source of more efficient new therapeutic anticancer drugs. In addition, recent trends in the management of cancer development include increasing awareness and chemoprevention that suggest using natural or synthetic chemicals to prevent initiation and promotional events associated with cancer development. Marine and terrestrial plants and animals are the main sources of natural products. They are considered as a fertile ground for finding novel antitumor drugs. Medicinal plants, used in folk medicine worldwide, are studied in ethnobotany and ethno-pharmacology. Up to the present date, more than 40 new cucurbitacins and cucurbitacin-derived compounds have been isolated from the cucurbitaceae family and from other species of the plant Kingdome. The most significant mechanisms with regard to the apoptotic effects of cucurbitacins are their ability to modify mitochondrial transmembrane potential and transcriptional activities via nuclear factors or genes and their capability to activate or inhibit pro- or antiapoptotic proteins. In general, cucurbitacins are considered to be selective inhibitors of the JAK/STAT pathways; however, other mechanisms may be implicated in their apoptotic effects, including the MAPK pathway (known to be important for cancer cell proliferation and survival), PARP cleavage, expression of active caspase-3, decreased pSTAT3 and JAK3 levels, as well as decreases in various downstream STAT3 targets such as Mcl-1, Bcl-2, Bcl-xL, and cyclin D3, all of which are implicated in apoptosis and the cell cycle control

\section{Physical properties and solubility of Cucurbitacins}

At room temperature, cucurbitacins are generally crystalline substances. The chemical structure of cucurbitacins reveals their possession of hydrophobic properties, and thus poor water solubility. (1) To date, only a limited number of polymeric micellar systems have shown positive results in tumor targeted delivery of poorly soluble drugs after systemic administration. (2) Polyethylene oxide block micelles are nanoscopic carriers $(20-100 \mathrm{~nm}$ in size) with a hydrophilic shell/hydrophobic core structure that have shown great promise in the solubility and controlled delivery of hydrophobic drugs. (3) Poly (ethylene oxide)block-poly ( $\varepsilon$-caprolactone) (PEO-b-PCL) and poly (ethylene oxide)-block-poly ( $\alpha$-benzyl carboxylate $\varepsilon$-caprolactone) (PEO-b-PBCL) micelles $(<90 \mathrm{~nm})$ were engineered by a cosolvent evaporation technique as nanocarriers for the delivery of cucurbitacins, which are inhibitors of the signal transducer and activator of transcription 3 (STAT3). It was determined that the anti-cancer and STAT3 inhibitory activity of the polymeric micellar cucurbitacins were comparable to the free drugs on a B16F10 melanoma cell line in vitro. Intra-tumoral injection of $1 \mathrm{mg} / \mathrm{kg} /$ day cucurbitacin I resulted in the regression of established B16F10 mouse melanoma tumors in vivo. In comparison to free cucurbitacin I, PEO-bPBCL micellar cucurbitacin I was found to provide comparable anti-cancer effects against B16-F10 tumors and limit drug levels in animal serum while maintaining high drug levels in tumor following intra-tumoral administration. The results indicate the potential of polymeric micelles as suitable vehicles for the delivery of cucurbitacins I and B. Interestingly, the toxicity associated with cucurbitacins I and B was significantly reduced when these drugs were loaded into the nanoparticles.

\section{Cucurbitacins, Stats and Tumerorigenesis}

Signal transducers and activators of transcription (STATs) are a family of seven proteins including STAT 1, 2, 3, 4, 5a, 5b, and 6. STAT3 protein is ubiquitously expressed in most tissues. (4) Each has its unique function to transduce extracellular signals and directly modulate transcription. STAT proteins play important role in regulation of immune response, inflammation, proliferation, differentiation, development, cell survival, and apoptosis. (5) STATs are initially present in inactive forms in the cytoplasm. Upon stimulation by a wide variety of receptormediated growth factors such as plateletderived growth factor (PDGF) or epidermal growth factor (EGF) (6) and cytokines such as interleukin-6 (IL-6) or interferon (7) signaling, STATs associate the cell membrane receptors to be activated via phosphorylation at conserved tyrosine 705 residues either directly 
by receptor tyrosine kinases, or indirectly by non-receptor tyrosine kinases, e.g., Janus kinases (JAKs) and Src oncogenic kinase. The JAKs then phosphorylation of tyrosine 705 induces STAT dimerization, nuclear translocation, and DNA binding at STATspecific sequence in the promoter regions of their target genes to stimulate their transcription. (8) Additionally, serine at the $727^{\text {th }}$ residue in the same domain must also be phosphorylated for complete transcriptional activity. (9) In normal physiological conditions, the activation duration of STAT protein especially STAT3 is temporary and strictly controlled illustrates the JAK/STAT pathway for activation and inhibition.

\section{Conclusion}

Cucurbitacin structures are characterized by the tetracyclic cucurbitane nucleus (triterpenes) with a variety of oxygen substitutions at different positions. Because of the possession of hydrophobic properties and poorly soluble water, polymeric micellar systems exhibited improved antitumor efficacy because of a better solubilization and targeting after local and / or systemic administration. Different cucurbitacin compounds exhibit antitumor proliferation inhibition and induce apoptosis alone or synergistically with other proven anticancer chemicals and cytokines using numerous human cancer cell lines and tumor xenografts of leukemia, lymphoma, breast, prostate, lung, uterine cervix, liver, skin, colon, laryngeal, brain and pancreatic cancers. In a structure-function related manner, cucurbitacins' inhibition of phosphorylation of STAT3 and/or JAK2 and their subsequent activation seamed as the major mechanism of their action.

\section{Acknowledgements}

I would like to express my gratitude to Himalayan Journal of Health Sciences who gave me the opportunity to publish the article.
Financial Disclosure statement: The author received no specific funding for this work.

\section{Conflict of Interest}

The author declares that there is no conflict of interest regarding the publication of this article.

\section{References}

1. Gry J, Søborg I, Andersson HC. Cucurbitacins in plant food. Vol. 556. Tema Nord Nordic Council of Ministers; Ekspressen Tryk \& Kopicenter; Copenhagen: Identity physical and chemical properties and analytical methods; 2006.p. 17-22.

2. Aliabadi HM, Lavasanifar A. Polymeric micelles for drug delivery. Expert Opin Drug Deliv. 2006; 3:139-62.

3. Kwon GK, Forrest ML. Amphiphilic block copolymer micelles for nanoscale drug delivery. Drug Dev Res. 2006; 67:15-22.

4. Molavi O, Ma Z, Mahmud A, Alshamsan A, Samuel J, Lai R, Kwon GS, Lavasanifar A. Polymeric micelles for the solubilization and delivery of STAT3 inhibitor cucurbitacins in solid tumors. Int J Pharmaceutics. 2008; 347:118-27.

5. Clericuzio M, Mella M, Vita-Finzi P, Zema M, Vidari G. Cucurbitane triterpenoids from Leucopaxillus gentianeus. J Nat Prod. 2004; 67:1823-8.

6. Recio MC, Prieto M, Bonucelli, Orsi C, Manez S, Giner RM, Cerda-Nicolas M, Rios JL. Antiinflammation activity of two cucurbitacins isolated from Cayaponia tayuya roots. Planta Med. 2004; 70:414-20.

7. Leaman DW, Pisharody S, Flickinger TW, Commane MA, Schlessinger J, Kerr IM. Roles of JAKs in activation of STATs and stimulation of cfos gene expression by epidermal growth factor. Mol Cell Biol. 1996; 16:369-75.

8. Hirano $T$, Nakajima $K$, Hibi M. Signaling mechanisms through gp130: a model of the cytokine system. Cytokine Growth Factor Rev. 1997; 8:241-52.

9. Stahl N, Farruggella TJ, Boulton TG, Zhong Z, Darnell JE, Jr, Yancopoulos GD. Choice of STATs and other substrates specified by modular tyrosinebased motifs in cytokine receptors. Science. 1995; 267:1349-53. 